\title{
Connecting Diffraction-Based Strain with Macroscopic Stresses in Laser Powder Bed Fused Ti-6Al-4V
}

\author{
TATIANA MISHUROVA, KATIA ARTZT, JAN HAUBRICH, SERGEI EVSEVLEEV, \\ ALEXANDER EVANS, MATTHIAS MEIXNER, ITZIAR SERRANO MUNOZ, \\ IGOR SEVOSTIANOV, GUILLERMO REQUENA, and GIOVANNI BRUNO
}

\begin{abstract}
The laser powder bed fusion (LPBF) production process often results in large residual stress (RS) in the parts. Nondestructive techniques to determine RS are badly needed. However, a reliable quantification of macro-RS (i.e., stress at the component level) by means of diffraction-based techniques is still a great challenge, because the link between diffraction-based strain and macro-RS is not trivial. In this study, we experimentally determine (by means of in-situ synchrotron radiation diffraction) this link for LPBF Ti-6Al-4V. We compare our results with commonly used models to determine the so-called diffraction elastic constants (DECs). We show that LPBF materials possess different DECs than wrought alloys, simply because their microstructural and mechanical properties are different. We also show that the existing models can be used to calculate DECs only if high accuracy of the RS values is not required. If the peculiarities of the microstructure have to be taken into account (as is the case of additively manufactured materials), a radically new approach is desirable.
\end{abstract}

https://doi.org/10.1007/s11661-020-05711-6

(C) The Author(s) 2020

\section{INTRODUCTION}

ONE of the most important issues in laser powder bed fusion (LPBF) additive manufacturing (AM) is the influence of residual stress (RS) on parts during production and service. ${ }^{[1]}$ High-temperature gradients and cooling rates during production often lead to tensile RS. ${ }^{[2-4]}$ Such stresses may reach values close to the yield

\footnotetext{
TATIANA MISHUROVA, SERGEI EVSEVLEEV, ALEXANDER EVANS, and ITZIAR SERRANO MUNOZ are with the Bundesanstalt für Materialforschung und -prüfung (BAM), Unter den Eichen 87, Berlin 12205, Germany. Contact email: tatiana.mishurova@bam.de KATIA ARTZT and JAN HAUBRICH are with the Institute of Materials Research, German Aerospace Center (DLR; Deutsches Zentrum für Luft -und Raumfahrt), Linder Höhe, Cologne 51147, Germany. MATTHIAS MEIXNER is with the Helmholtz-Zentrum Berlin für Materialien und Energie GmbH, HahnMeitner Platz 1, 14109, Berlin, Germany. IGOR SEVOSTIANOV is with the Department of Mechanical and Aerospace Engineering, New Mexico State University, Las Cruces, NM 88003-8001. GUILLERMO REQUENA is with the Institute of Materials Research, German Aerospace Center (DLR; Deutsches Zentrum für Luft -und Raumfahrt), Linder Höhe, and the Metallic Structures and Materials Systems for Aerospace Engineering, RWTH Aachen University, 52062 Aachen, Germany. GIOVANNI BRUNO is with the Bundesanstalt für Materialforschung und -prüfung (BAM), Unter den Eichen 87, and the Institute of Physics and Astronomy, University of Potsdam, KarlLiebknecht-Straße 24/25, Potsdam 14476, Germany.

Manuscript submitted September 26, 2019.
}

Article published online March 27, 2020 limit in the subsurface region, ${ }^{[5,6]}$ thereby decreasing the performance of the material, especially during cyclic loading. Moreover, RS can induce severe geometrical distortions and cracking, ${ }^{[4]}$ thus nullifying the main advantage of freeform fabrication. Therefore, quantification of RS and validation of thermomechanical models by experimental results are among the greatest challenges for qualification of AM parts.

The nondestructive (diffraction-based) experimental determination of $\mathrm{RS}$ is directly connected to the microstructure of the part. The complicated thermal history and high anisotropy of LPBF parts lead to microstructures and mechanical properties different from conventionally manufactured materials (wrought or cast). ${ }^{[7]}$ The microstructure in LPBF materials is additionally highly dependent on process parameters. ${ }^{[8-10]}$ Therefore, the elastic properties may differ from conventional materials and even vary with the production parameters of LPBF. The knowledge about elastic properties is important for RS analysis by diffraction techniques, where the so-called diffraction elastic constants (DECs) are used ${ }^{[11]}$ to connect the measured microstrain (diffraction based, i.e., averaged over grains of a particular family) with the macrostress (at the component length scale). Therefore, the use of incorrect DECs can lead to erroneous RS values ${ }^{[12]}$ and misestimation of the material performance. 
In-situ synchrotron X-ray diffraction (SXRD) or neutron diffraction (ND) is particularly suited for the experimental determination of DECs. ${ }^{\text {13,14] }}$ However, the available literature lacks information about the DECs of AM materials, with the consequence that values only valid for cast or wrought alloys are used for RS determination or modeling. Recently, in-situ tensile tests with SXRD have been reported on Ti-6Al-4V LPBF samples in as-built condition, targeted at studying the effect of different build orientations, ${ }^{[15]}$ but the DECs have not been discussed. Dumontet et al. ${ }^{[16]}$ first attempted evaluating the DEC of martensitic $\alpha^{\prime}$ LPBF Ti-6Al-4V material and found a difference of DECs up to 32 pct between pure $\alpha-\mathrm{Ti}$ and $\alpha^{\prime}-\mathrm{Ti}$ in LPBF Ti-6Al-4V.

It has been shown that the experimentally determined DECs of wrought $\mathrm{Ti}$ alloys differ from theoretical values. ${ }^{[14]}$ One of the reasons is the influence of the alloy composition, because some theoretical models consider single-crystal elastic constants of pure Ti. Indeed, the authors of Reference 12 have found a discrepancy between modeled and experimentally determined DECs for $\mathrm{Ti}-2.5 \mathrm{Cu}$ alloy and explained it by the influence of the $\mathrm{Cu}$ addition. The second reason of the discrepancy between theory and experiment is the influence of the specific microstructure and of the crystallographic texture (i.e., anisotropy). In Reference 17, a difference of lattice strain evolution between a unidirectionally rolled plate and some forged bar samples has been reported and ascribed to the presence of texture. LPBF materials are generally highly textured owing to the temperature gradients across the deposited layers (such gradients induce epitaxial growth along the building direction). ${ }^{[18-21]}$ Also, the change of microstructure by means of heat treatment and its influence on the elastic behavior of grains is not captured by classic theoretical models, which are mostly used for RS calculation (e.g., Voigt, Reuss, and Kröner). ${ }^{[22]}$ Much work has been conducted to improve those classic schemes for calculation of DECs, ${ }^{[23,24]}$ and recently, some work to calculate single-crystal elastic constants ${ }^{[25,26]}$ from in-situ experiments, taking into account the particular microstructural features (such as grain size and crystallographic texture). A detailed discussion of such models is out of the scope of this work and will be presented in a companion publication. Here, we focus on the experimental determination of DECs of LBPF Ti-6Al-4V as a tool to understand the elastic anisotropy of AM materials and the use of the appropriate assumptions for modeling. Such DECs can then be directly used for RS determination of that particular material.

We performed in-situ SXRD investigations on specimens in as-built conditions and after heat treatment. The link between the diffraction (micro-)strain response to an externally applied macrostress was rationalized in terms of a stress-microstrain conversion tensor $(S \mu E C T)$, as used in micromechanics. We show that such a tensor generalizes the concept of DECs and allows a critical discussion on the validity of the approximations made in the current models.

\section{EXPERIMENTAL}

\section{A. Material}

Ti-6Al-4V rods ( $8 \mathrm{~mm}$ in diameter, $95 \mathrm{~mm}$ in length) and cuboids (with size $5 \times 5 \times 15 \mathrm{~mm}^{3}$ ) were printed on a steel base plate (preheated to $200{ }^{\circ} \mathrm{C}$ ) with the use of support structures and with their long axis along the build direction. An SLM Solutions $280^{\mathrm{HL}}$ machine was employed, using plasma-atomized Ti-6Al-4V ELI grade 23 powder from AP\&C with a particle size of $d_{90}<50$ $\mu \mathrm{m}$. The characterization of the powder has been reported elsewhere. ${ }^{[27]}$ The production parameters were set to the following: laser power of $175 \mathrm{~W}$, laser velocity of $500 \mathrm{~mm} / \mathrm{s}$, hatch distance of $100 \mu \mathrm{m}$, and layer thickness of $30 \mu \mathrm{m}$ (this parameter set is named A4 in our previous studies $\left.{ }^{[10,28]}\right)$. These manufacturing conditions were optimized in order to achieve minimum porosity and acceptable $\mathrm{RS}$ values. ${ }^{[10,29]}$ The chess scanning strategy was used with a field size of $5 \mathrm{~mm}$ and rotating $90 \mathrm{deg}$ from layer to layer.

Two samples were instigated in as-built conditions (labeled AB) and two samples were subjected to heat treatment at $530{ }^{\circ} \mathrm{C}$ for 2 hours after production (labeled 530C). The heat treatment was performed under Argon atmosphere with heating and cooling rate around $7{ }^{\circ} \mathrm{C} / \mathrm{min}$. For the in-situ mechanical tests, compression (_c) and tensile (_t) specimens were machined by turning without additional surface finishing applied (the geometry and dimensions are shown in Figure 1(a)).

\section{B. In-situ $S X R D$}

In-situ SXRD tension and compression tests were performed in the transmission mode at the EDDI beamline (BESSY II, HZB, Berlin). ${ }^{[30]}$ The EDDI diffractometer was operated in the energy-dispersive (ED) mode; therefore, the reflections of several crystallographic planes could be simultaneously tracked during the in-situ experiments. The diffraction peaks were fitted using a Pseudo-Voigt function; an in-house developed Mathematica code was employed. A liquid-nitrogen cooled Ge solid-state detector from Canberra (model GL0110) was used. The counting time was 2 minutes per spectrum. The aperture sizes of the primary and the secondary slits were $1 \mathrm{~mm} \times 1 \mathrm{~mm}$ and $100 \mu \mathrm{m} \times 5 \mathrm{~mm}$ (equatorial $\times$ axial), respectively. This allowed averaging across the entire sample diameter. For the in-situ mechanical tests, a load rig (Walter + Bai AG) with maximum load of $20 \mathrm{kN}$, mounted on an Eulerian cradle, was employed (Figure 1(b)). The building direction of the sample corresponded to the load direction during uniaxial tests (axis $X$, Figure 1(a)). Acquisitions were performed in axial and radial directions for every load step ( $\varepsilon_{\mathrm{ax}}$ and $\varepsilon_{\text {rad }}$ in Figure 1(a)), but for the sake of simplicity, only the axial data will be reported (see below). The load control mode was used with a loading rate of $10 \mathrm{~N} / \mathrm{s}$ between each counting step. The displacement was defined by the relative displacement of the clamps. It should be noticed that the in-situ tensile 

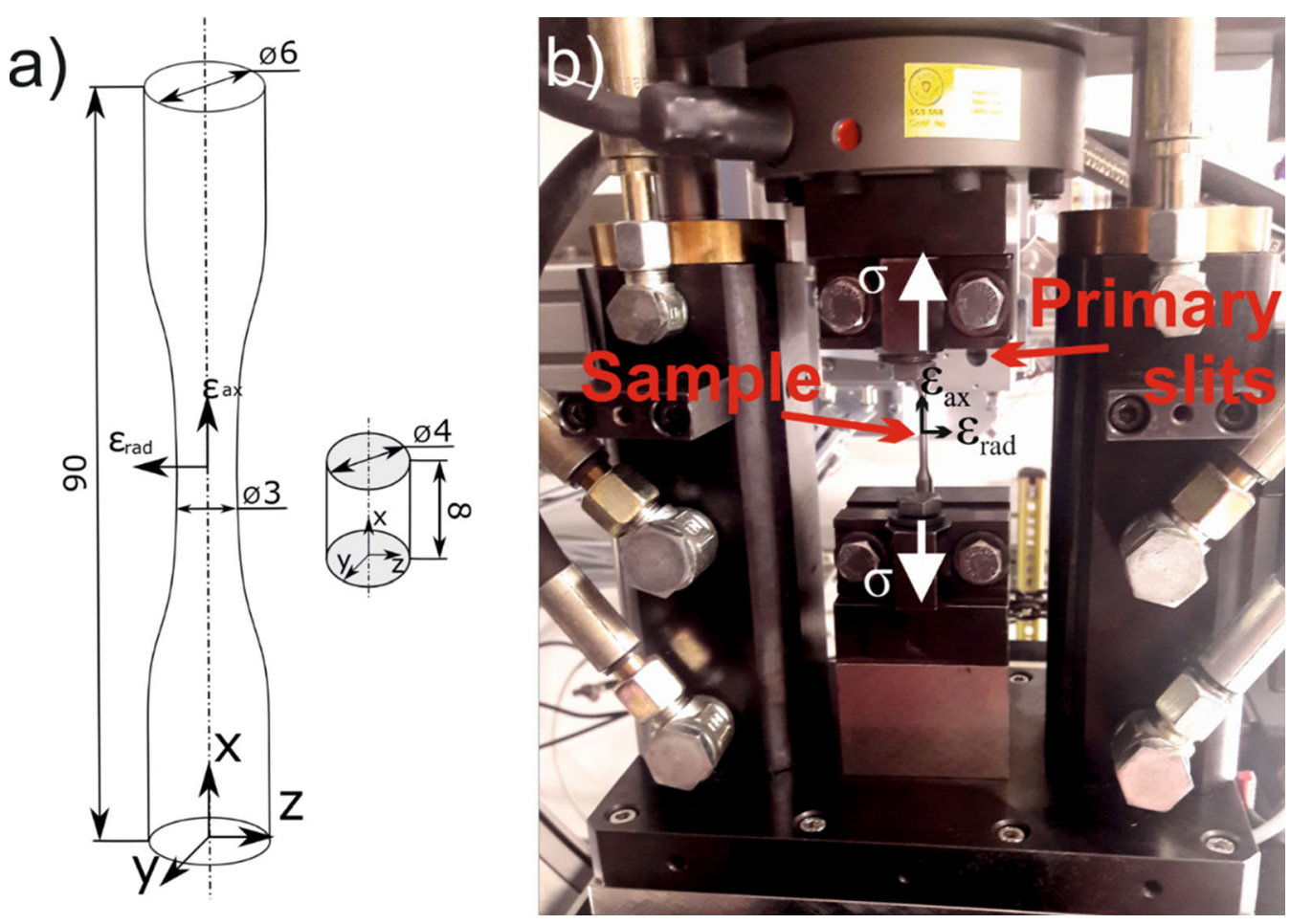

Fig. 1-(a) Sketch of tension (_t) and compression (_c) specimens with their coordinate system (all dimensions are in millimeters). (b) Picture of the $i n$-situ setup with a tensile sample mounted in the load rig (to measure $\varepsilon_{\mathrm{ax}}, \psi=0 \mathrm{deg}$ ).

tests were performed until failure, but only the elastic part is reported in the present study.

The lattice strain $\varepsilon^{h k l}$ was calculated from $d^{h k l}$ values at every load step as

$$
\varepsilon^{h k l}=\frac{d^{h k l}-d_{\mathrm{in}}^{h k l}}{d_{\mathrm{in}}^{h k l}}
$$

where $d_{\text {in }}^{h k l}$ was the lattice spacing for crystallographic plane families $\{h k l\}$ at preload (around $10 \mathrm{~N}$ ).

\section{Microscopy and Texture Analysis}

The microstructure was characterized in the clamp region at around $65 \mathrm{~mm}$ from the bottom of the sample. The measurements were performed in the plane perpendicular to the build direction. For microstructural analysis, $\mathrm{AB}_{-} \mathrm{t}$ and $530 \mathrm{C}_{-} \mathrm{t}$ samples were ground and then polished with an aqueous suspension of $0.04-\mu \mathrm{m}$ $\mathrm{SiO}_{2}$ particles with the addition of 10 pet $\mathrm{H}_{2} \mathrm{O}_{2}$. Scanning electron microscopy (SEM) was conducted in backscattered electron mode (BSE) on a LEO 1530VP microscope with Gemini tower (Zeiss). The operation voltage was $15 \mathrm{kV}$ and the working distance $7 \mathrm{~mm}$.

Texture analysis was performed in the ED diffraction mode, employing the ED laboratory diffractometer LEDDI (HZB, Berlin). LEDDI features are described in detail in Reference 31. The experiments were conducted in reflection geometry. The texture measurements were performed in the plane perpendicular to the build direction, at the same location where microscopy was made: around $65 \mathrm{~mm}$ from the bottom of the sample and in the center of the sample. Only one location per sample was considered, since no variation of the microstructure was observed. A conventional tungsten long fine-focus X-ray tube was operated at $U$ $=60 \mathrm{kV}$ and $I=45 \mathrm{~mA}$ in combination with an ED detector system. The latter allows the simultaneous collection of multiple diffraction lines $h k l$ up to photon energies of $60 \mathrm{keV}$ in a single spectrum recorded under an arbitrary but fixed Bragg angle $2 \theta$. For texture analysis, ED spectra were acquired at azimuthal angles $\varphi$ (rotation in the $Y Z$ plane) and the inclination angles $\psi$ (tilting in $X Z$ plane) in the intervals from $\varphi \in[0 \mathrm{deg} ; 355$ $\mathrm{deg}]$ and $\psi \epsilon$ [0 deg; $85 \mathrm{deg}]$ in steps of $5 \mathrm{deg}$. Overall, six $h k l$ reflections of $\alpha / \alpha^{\prime}-\mathrm{Ti}$ could be detected $(002,100$, $101,102,103$, and 110) and were evaluated in order to determine the orientation distribution function (ODF). From the ODF, pole figures for all crystallographic families $\{h k l\}$ could be calculated. The ODF as well as the recalculated pole figures were processed using the software package LaboTex 3.0. ${ }^{[32]}$

\section{X-ray Computed Tomography}

X-ray computed tomography (CT) was performed in the gage region (middle height of the sample) prior to the mechanical test of both tensile samples (AB $t$ and 530C t). A vitome $\mid x$ L 300 CT scanner from General Electric (GE) was used. 2000 projections were acquired for each CT scan. A tube voltage of $125 \mathrm{kV}$ and a current of $60 \mu \mathrm{A}$ were used. The acquisition time for each projection was 2 seconds. The GE standard commercial reconstruction algorithm was used and the 
beam hardening correction was selected based on reconstructed images. A voxel size of $(4 \mu \mathrm{m})^{3}$ was achieved. Data processing was performed using the AvizoFire 9.4 software package. ${ }^{[33]}$ The pores were identified by global threshold segmentation. To remove noise, raw data were filtered by a non-local means filter, implemented in the AvizoFire 9.4. software. For the calculation of the volume fraction of pores, only pores larger than eight voxels were considered to reduce the probability of false segmentation.

\section{RESULTS}

The microstructure of the AB sample presents $\alpha+\alpha^{\prime}$ laths (Figure 2(a)); this is typical for Ti-6Al-4V LPBF in the as-manufactured condition. The 530C sample shows a small amount of $\beta$-precipitates nucleated at the grain boundaries of $\alpha$ lamellae, as also shown in References 9 and 34. Haubrich et al. ${ }^{[9]}$ reported that the mass fraction of $\beta$-Ti phase reaches around 3 pct. This heat treatment was adopted as it is supposed to be stress relieving without inducing large microstructural modifications.

The microstructure, presented in Figure 2, is assumed to be representative of the gage volume used in the in-situ experiments. In fact, Barriobero-Vila et al. ${ }^{[35]}$ showed that only a few last building layers (i.e., within the last millimeter of the built) have different microstructures (more precisely, $\beta$-phase content) from the rest of the sample in LPBF Ti-6Al-4V. Additionally, the gage volume used in the synchrotron radiation diffraction experiments practically covers the entire sample diameter $(3 \mathrm{~mm})$, thereby averaging the local microstructural variations.

From the three-dimensional reconstructions of the volumes and rendering of defects, projections along the $X$-axis and $Y$-axis could be extracted. They show the spatial distribution of defects (Figure 3). Lack of fusion defects, ${ }^{[29]}$ typical for LPBF, and round gas pores could be observed in both samples. As mentioned above, the manufacturing conditions were considered as porosity optimized (reported in our previous studies). ${ }^{[10,29]}$ Therefore, the investigated samples were expected to be close to fully dense. Indeed, the analysis of the volume fraction of pores (for the part of tensile samples) showed 0.01 vol pet for both AB_t and 530C_t samples (Figure 3). The alignment and the location of pores in the build plane $(Y Z)$, as well as the complex shape of lack of fusion defects, can be crucial for the mechanical behavior even if the overall porosity is small. ${ }^{[36]}$ However, in our case, we can safely assume that they do not play a significant role in the mechanical behavior.

The diffraction patterns obtained during the in-situ experiments in axial and radial directions for $\mathrm{AB}$. $\mathrm{t}$ and 530C_t samples are presented in Figure 4. Their difference gives a footprint of the preferential crystallographic orientation. For the analysis of the elastic response, only reflections present in both directions were considered (indexed in Figures 4(a) and (c)). As expected from the microstructure (Figure 2), a small difference between $A B \_t$ and 530C_t samples is visible (see the presence of the 112-reflection in the axial direction in Figure 4(a)), which is due to the small difference in $\beta$-phase content (Figure 4(d)). It should be noticed that the $\beta-200$ reflection was observed for the $530 \mathrm{C}_{-} \mathrm{t}$ samples only in one direction and with low intensity; therefore, it will not be discussed in the remainder of the article.

The pole figures for nine crystallographic planes of $\alpha-\mathrm{Ti}$, calculated from ODF of the AB_t sample, are presented in Figure 5. The as-built and the heat-treated materials show similar texture (Figure 4) so that the texture for the heat-treated material is not reported for the sake of brevity. The pole figures indicate a double fiber texture (with fiber axes 102 and 110), and therefore a transversely isotropic behavior.

For RS determination in engineering components, in most of the cases, the assumption of quasi-isotropy is made and, therefore, only two DECs are used. Therefore, the elastic responses along the axial and radial directions are the most relevant for experimentalists. It is widely reported that the yield stress of AB LPBF Ti-6Al-4V reaches around 900 to $1100 \mathrm{MPa}{ }^{[37]}$ Therefore, in order to obtain the DEC for each crystallographic family, only the elastic part (up to $600 \mathrm{MPa}$ ) of the applied stress vs microscopic strain was analyzed (Figure 6). In the radial direction of the AB_t sample, a mismatch between the start point (unstrained) and the first loading point can be observed (Figure 6(a)): For almost all reflections, a tensile strain is recorded. This behavior is not observed for the heat-treated $530 \mathrm{C}$
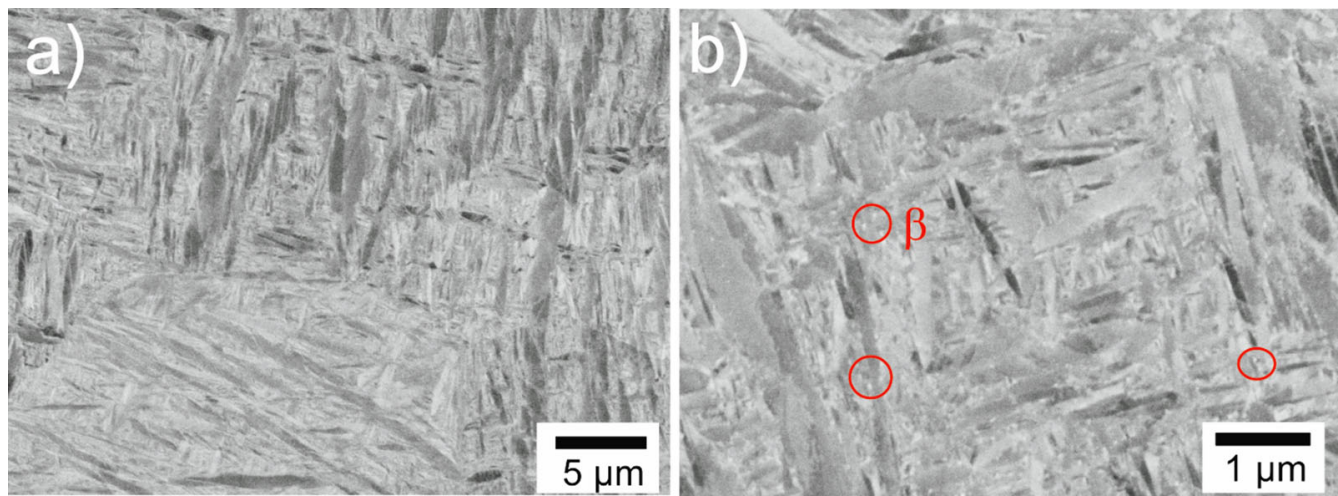

Fig. 2-BSE-SEM image taken in the build plane for $(a)$ AB_t and (b) 530C_t. Red circles show examples of $\beta$-Ti particles. 


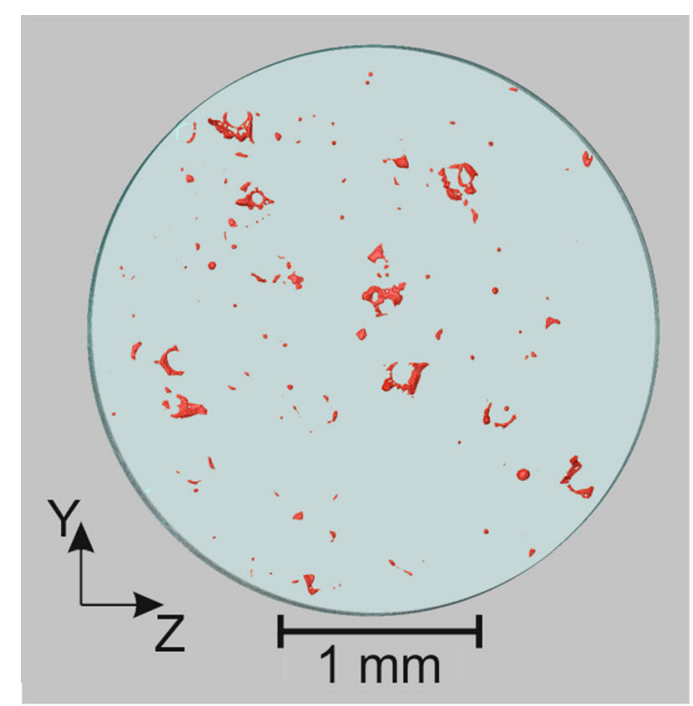

(a)

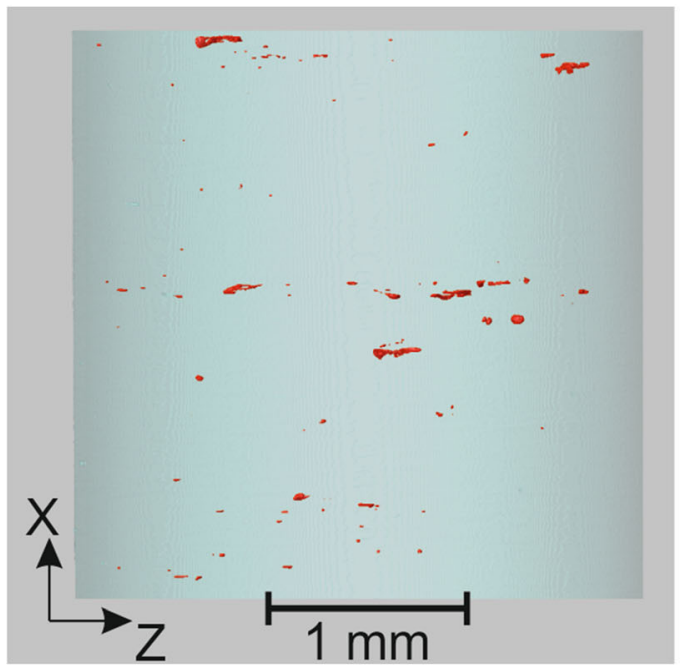

(c)

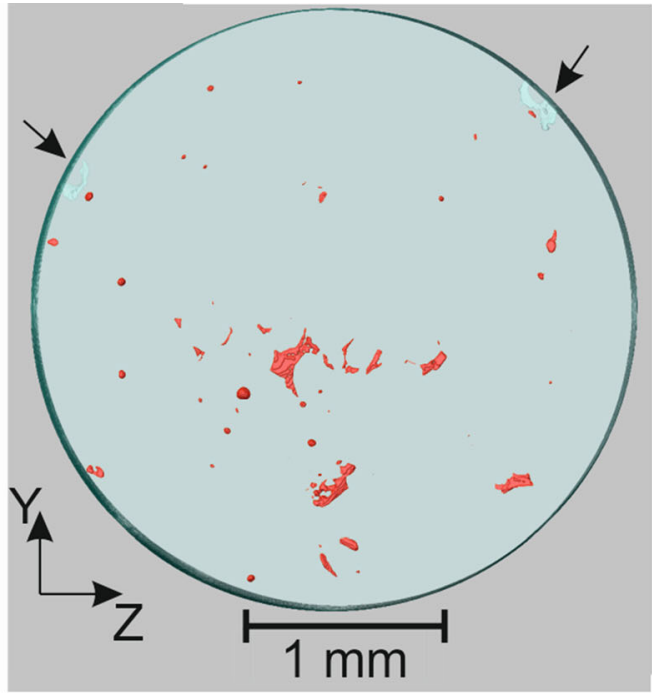

(b)

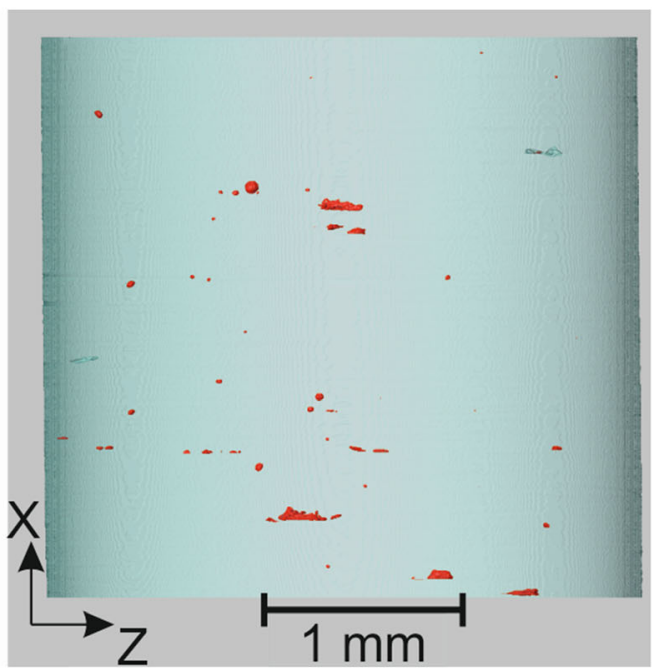

(d)

Fig. 3-Three-dimensional rendering of pores (in red) projected onto the $Y Z$ plane $(a)$ AB_t and (b) 530C_t and onto the $X Z$ plane for $(c)$ AB_t and (d) 530C_t. Pores open to the surface are shown with arrows.

sample (Figure 6(b)). Therefore, it could be caused by a small amount of RS, ${ }^{[5]}$ which relaxed after the first load step. The shift could be explained also by some initial misalignment of the AB_t sample, successively disappearing as the load is applied. For higher loads, the deformation has a linear behavior as a function of applied stress. Sample AB_c does not present such a behavior. For the 530C_t sample along the axial direction, the two secondary prismatic planes $(\{210\}$ and $\{110\})$ show the lowest slope of the applied stress-microstrain curve. Also, the elastic anisotropy is more pronounced for the $530 \mathrm{C}_{-} \mathrm{t}$ as compared to the $\mathrm{AB} \mathrm{B}$ specimen. In contrast, $530 \mathrm{C}_{-} \mathrm{c}$ shows almost no scatter among different crystallographic planes along the axial direction but some anisotropy in the radial direction.

Due to texture, not every diffraction peak from the pole figures of Figure 5 was observed during the in-situ experiments. For example, no grains of the $\{002\}$ and $\{100\}$ families were found to be oriented along the build direction (Figure 5, $\varphi=0 ; \psi=0$ ); i.e., they were not detectable in the in-situ experiment. Moreover, only low intensities for 103 and 101 reflections were detected. Therefore, it was not possible to directly analyze the mechanical response to external stress of basal and first-order prismatic planes.

The connection between diffraction-based microstrain and engineering macrostress is often made by DEC. ${ }^{[11]}$ However, such a concept exists in more general form in micromechanics, ${ }^{[38]}$ where DEC can be viewed as a component of the product of the strain concentration tensor $\Theta_{i j m n}$ and the tensor of macroscopic elastic compliances $S_{m n k l}$ so that $A_{i j k l}=\Theta_{i j m n} S_{m n k l}$. The link between microstrain and macrostress becomes, in such formalism, 


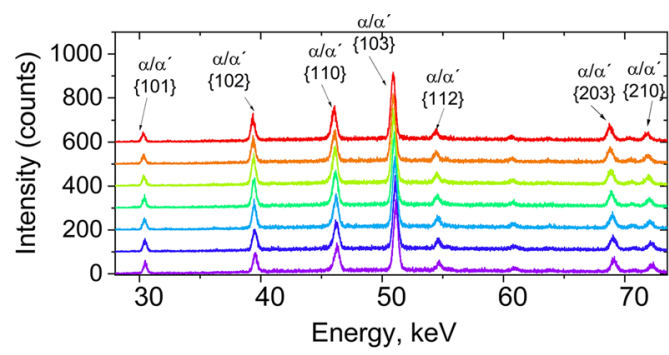

(a)

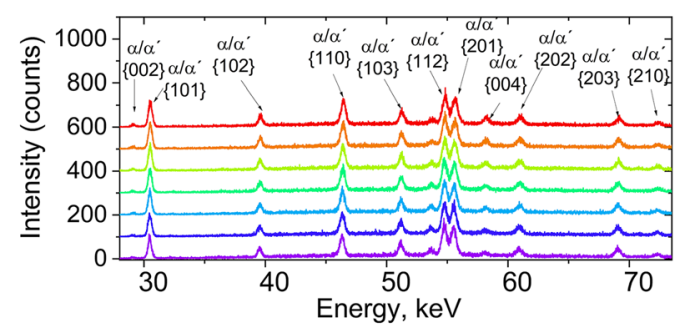

(b)

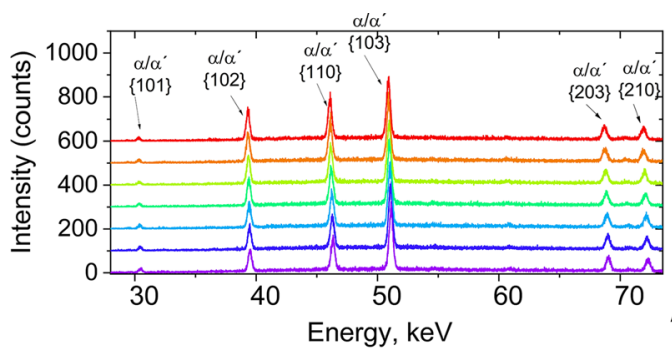

(c)

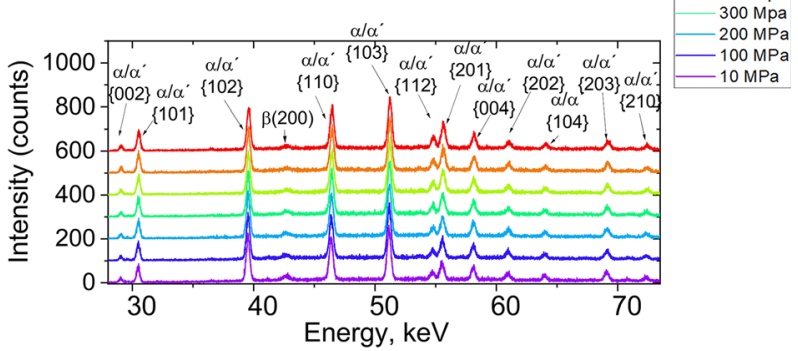

(d)

Fig. 4-Diffraction patterns obtained during in-situ experiment for sample AB_t $(a)$ in the axial direction and $(b)$ in the radial direction and for sample 530 C_t $(c)$ in the axial direction and $(d)$ in the radial direction. Note: Only the reflections present in both directions (indexed in plots (a) and (c)) were considered for further analysis.

$$
\left\langle\varepsilon_{i j}\right\rangle_{\text {micro }}=\left\langle A_{i j k l}>\sigma_{k l}\right.
$$

We introduce the tensor $A_{i j k l}$ as a $S \mu E C T$. It connects the elastic behavior of an individual grain with the effective (macroscopic) elastic properties of the specimen (material surrounding the grain). Importantly, $S \mu E C T$ also depends on the morphology of the grains - their shapes and orientations. Such an aspect is usually not considered by models developed to calculate DEC. In Eq. [2], the tensor $A$ is averaged because it is classically defined pointwise, but here, it is applied to the entire gage volume (under the assumption that the diffracting grains represent the gage volume).

In our case, we recorded the axial and transverse response (microstrain) to a uniaxial applied stress, and Eq. [2] rewrites

$$
\begin{aligned}
& \left\langle\varepsilon_{a x}^{h k l}\right\rangle_{\text {micro }}=\left\langle\varepsilon_{x x}\right\rangle_{\text {micro }}=<A_{x x x x}>\sigma_{x x}=<A_{\mathrm{ax}}^{h k l}>\sigma_{\mathrm{app}} \\
& \left\langle\varepsilon_{\mathrm{rad}}^{h k l}\right\rangle_{\text {micro }}=\left\langle\varepsilon_{z z}\right\rangle_{\text {micro }}=\left\langle A_{x x z z}>\sigma_{x x}=<A_{\mathrm{rad}}^{h k l}>\sigma_{\mathrm{app}}\right.
\end{aligned}
$$

where both the strains and the $A_{i j k l}$ tensor components are $h k l$ dependent. By fitting the experimental data $d_{\mathrm{ax}}^{h k l}$ or $d_{\text {rad }}^{h k l} v s \sigma_{\text {app }}$ with a linear function, the $S \mu E C T$ component $A_{\mathrm{ax}}^{h k l}$ in the loading direction or $A_{\mathrm{rad}}^{h k l}$ transverse to it can be calculated by, e.g.,

$$
d_{\mathrm{ax}}^{h k l}=d_{\mathrm{in}}^{h k l} A_{\mathrm{ax}}^{h k l} \sigma_{\mathrm{app}}+d_{\mathrm{in}}^{h k l}
$$

It must be kept in mind, however, that $A_{\text {rad }}^{h k l}$ represents different grains than $A_{\mathrm{ax}}^{h k l}$ (the reflection $h k l$ is generally not caused by the same grains in two perpendicular directions unless very particular reflections are considered). Moreover, due to texture, the response in the radial direction of the same grain can be different from the axial (different constraints from surrounding grains). We will, therefore, focus on the axial response only.

\section{DISCUSSION}

In the literature, it is common to indicate the inverse of $S \mu E C T$ components (e.g., $A_{\mathrm{ax}}^{-1}$ ) as DEC and calculate them by means of commercial or in-house software, based on Voigt, Reuss, and Kröner schemes. ${ }^{\left[{ }^{39]}\right.}$ In these models, two assumptions are commonly made: (1) the material is macroscopically isotropic (but individual grains can be anisotropic) and (2) grains are perfectly randomly oriented. Kröner's model further assumes that grains are spherical. In many structural materials, these conditions may not be fully satisfied and, in fact, some improvements of classic schemes have been proposed to take into account texture and microstructural features. ${ }^{[23,26,40]}$ However, the simplest versions of such models are still widely used by experimentalists for RS calculation, since full information about the material (such as grain morphology and size, texture, and anisotropy) is not always available. By comparing the output of such models with the experimental values of $A_{\text {ax }}^{-1}$, we can estimate the error associated to the usage of the common approximations for the calculation of RS. Therefore, we calculated the theoretical $A_{\mathrm{ax}}^{-1}$ values for $\alpha$-Ti (hcp) using the software XEC ${ }^{[41]}$ (Figure 7). When calculating $A_{\mathrm{ax}}^{-1}$, it is common to introduce the orientation parameter $H^{2}$. It defines the direction cosine of the angle between the prismatic $\{100\}$ and the basal $\{002\}$ planes, according to the formula 

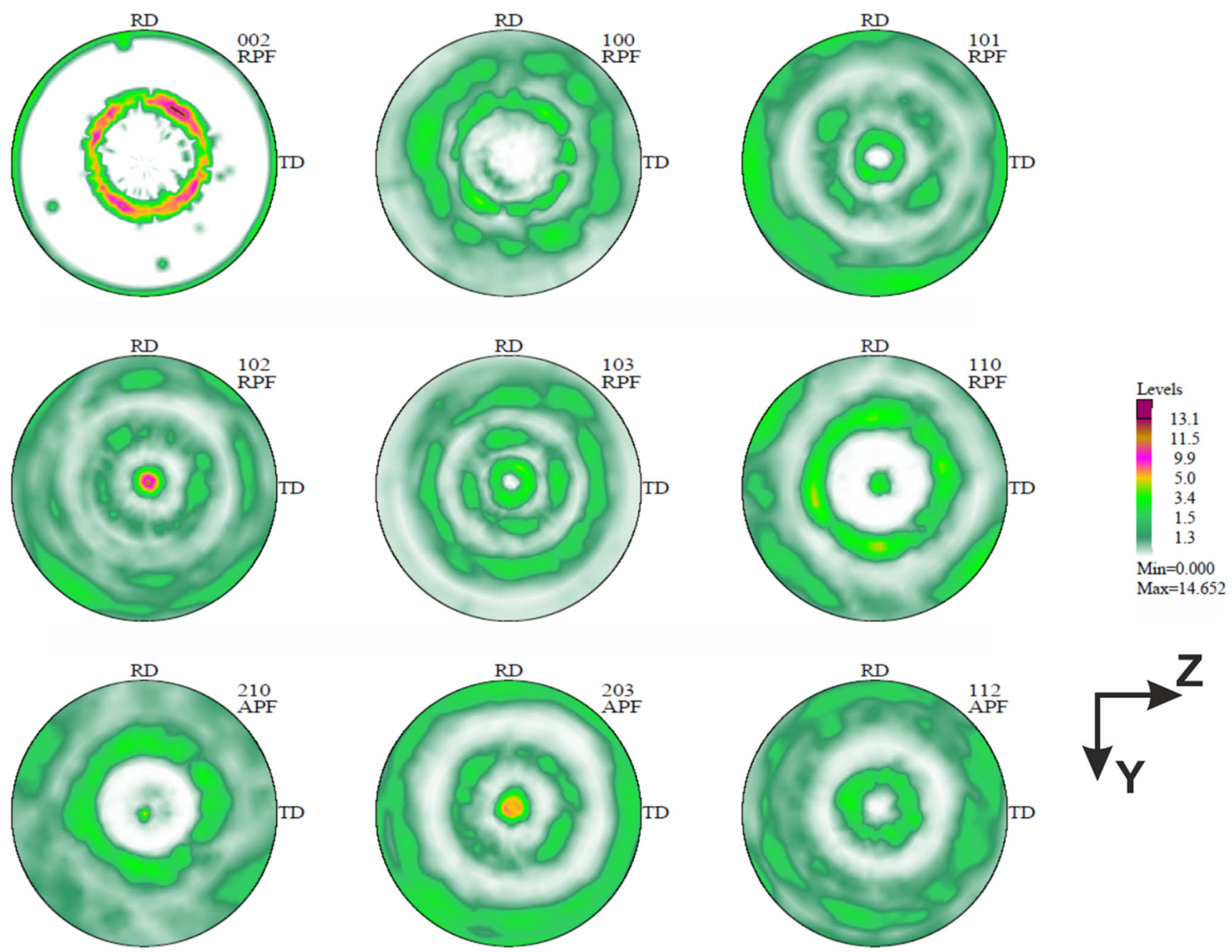

Fig. 5-Calculated pole figures for $\alpha$-Ti for the AB_t specimen.

$$
H^{2}=\frac{l^{2}}{\frac{4}{3}\left(\frac{c}{a}\right)^{2}\left(h^{2}+k^{2}+h k\right)+l^{2}}
$$

As discussed in References 39 and 42, Voigt's approximation presents no directional dependence of the DEC (on $h k l$ ): the inverse of $S \mu E C T$ axial component would get the value $A_{\mathrm{ax}}^{-1}=117 \mathrm{GPa}$ for all reflections (Figure 7). This causes a difference to experimental values up to $20 \mathrm{GPa}$ (i.e., 15 pct of the experimental values, Figure 7). Voigt's model yields indeed an estimation of the macroscopic elastic modulus. In fact, Simonelli et al. experimentally obtained a Young's modulus of $115 \pm 6 \mathrm{GPa}$ for Ti-6Al-4V LPBF samples built vertically. ${ }^{[4]}$ Voigt's scheme in this form is not suited for the calculation of $A_{\mathrm{ax}}^{-1}$ in diffraction-based RS analysis. It would be equivalent to the use of macroscopic elastic constants (as in Reference 44) for the link between microstrains and macrostresses. This should be avoided, especially in the case of strong anisotropy as commonly linked to materials produced by additive manufacturing processes such as LPBF.
Kröner's scheme captures the experimental values of $A_{\mathrm{ax}}^{-1}$ for low Miller indices (or $H^{2}$ parameters), corresponding to prismatic planes, while the Reuss model yields underestimated DECs. Consistently, for high orientation parameters (corresponding to pyramidal planes), the Reuss model captures the experimental values, while Kröner's model follows the lower bound of the experimental values (Figure 7). Relevantly, all theoretical schemes capture the experimental values of DEC for LPBF Ti-6Al-4V more precisely than the values reported for conventional Ti-6Al-4V (References 14 and 17). The $A_{\text {ax }}^{-1}$ difference between conventionally produced and AM Ti-6Al-4V reaches up to $20 \mathrm{GPa}$ (Figure 7). Similar findings have been reported in Reference 16. This effect can be attributed to the different texture and microstructure between the two materials. As mentioned previously, the $S \mu E C T$ automatically includes information about the microstructure (grain morphology and texture). Those two factors are very different for LPBF (martensitic $\alpha / \alpha^{\prime}$, Figure 2) and typical forged/wrought Ti-6Al-4V $(\alpha+\beta$, Reference 17). In order to capture all effects of texture and microstructure of LPBF materials, more sophisticated 


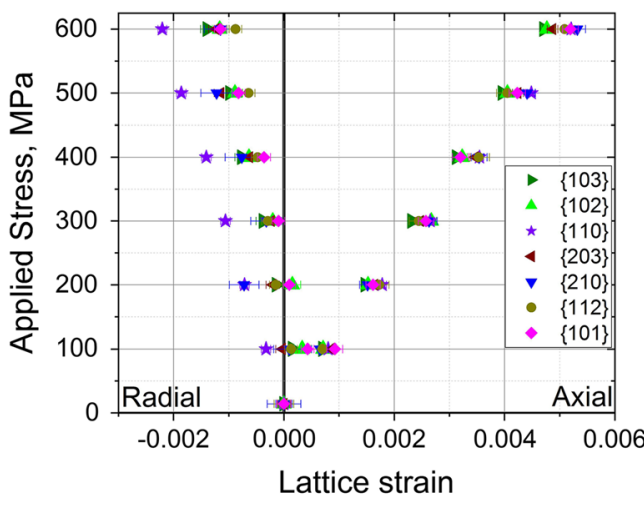

(a)

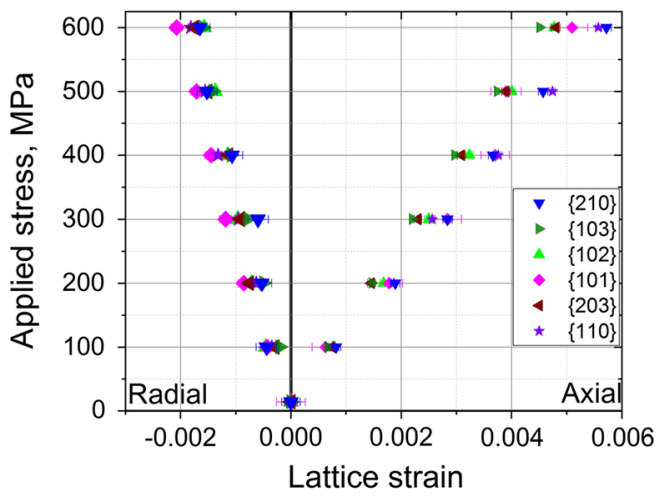

(b)

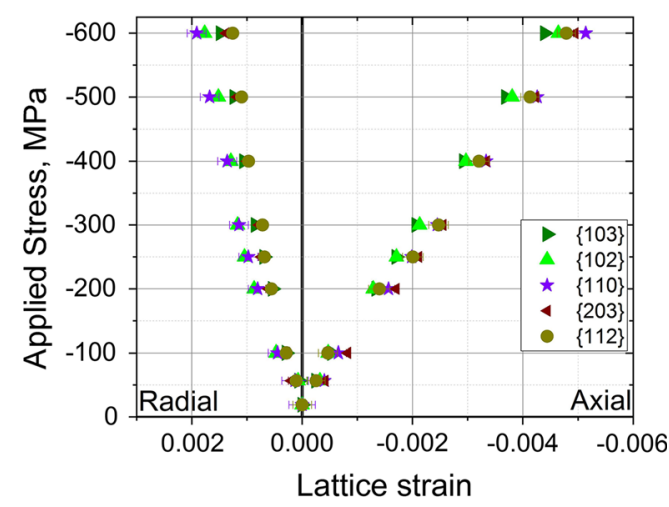

(c)

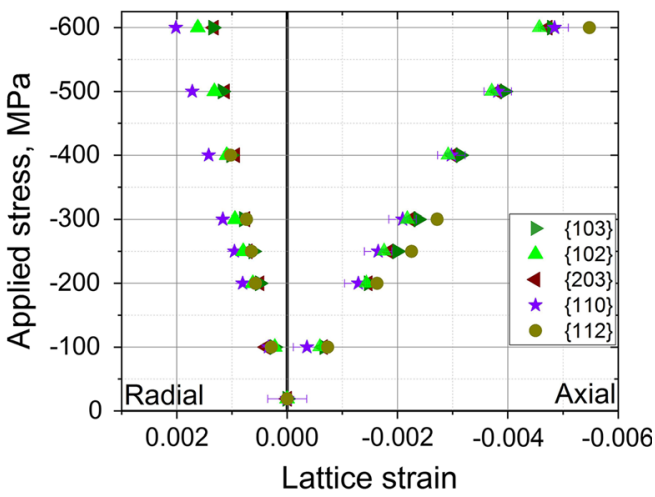

(d)

Fig. 6-Lattice strain evolution for different crystallographic planes during in-situ test for $(a)$ AB_t and (b) 530C_t (tension) and compression test for $(c) \mathrm{AB}_{-} \mathrm{c}$ and $(d)$ 530C_c (compression). Note the sign of the applied stress and the lattice strain.

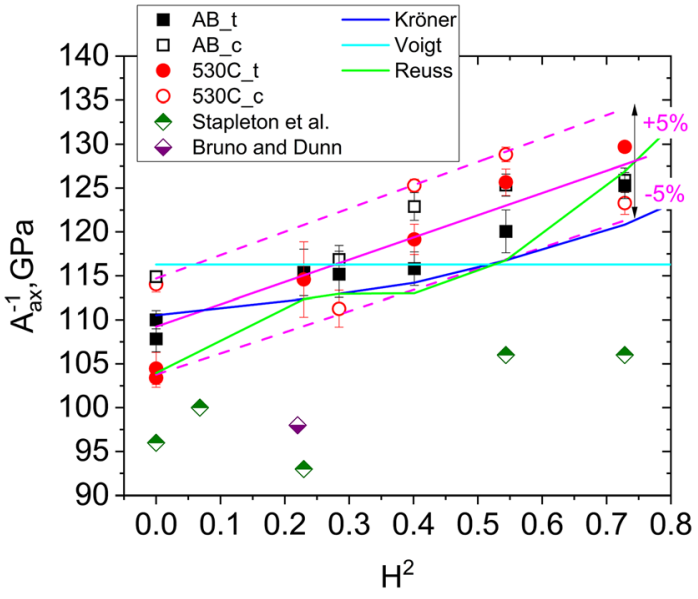

Fig. 7-The inverse of $S \mu E C T$ component along the axial direction $A_{\mathrm{ax}}^{-1}$ as a function of orientation factor $H^{2}$, as measured (points) and calculated (lines). The magenta lines show the linear fit of the experimental date and \pm 5 pct scatter around the mean value. Literature values for conventional Ti-6Al-4V are also reported, taken from Refs. ${ }^{[14]}$ and ${ }^{[17]}$

theoretical approaches should be used, ${ }^{[23,24,45]}$ moving beyond the classic schemes presented previously. This will be the subject of a companion article.

All experimentally determined $A_{\mathrm{ax}}^{-1}$ values lie in an interval \pm 5 pct from a linear fit of all datapoints (magenta line, Figure 7). The DEC values calculated by the Kröner and Reuss models are also in the range of the experimental scatter. Thus, if a high precision is not required for RS calculations, these models can be used for the calculation of $A_{\mathrm{ax}}^{-1}$ in LPBF Ti-6Al-4V as a first approximation. We observe that the precision can be even improved by considering low or high $H^{2}$ (Reuss model agrees with the data for low $H^{2}$, Kröner's for high $H^{2}$ ). However, $A_{\text {ax }}^{-1}$ of each single specimen has an experimental scatter of less than \pm 5 pct. Therefore, for a precise calculation of $\mathrm{RS}$, the $A_{\mathrm{ax}}^{-1}$ of the specific condition (tension/compression or heat treatment) should be taken.

In fact, the inverse of $S \mu E C T$ component $A_{\mathrm{ax}}^{-1}$ for compression specimens appears almost systematically larger than that for tensile (Figures 8(a) and (b)). The two sets of samples (with and without heat treatment) show a similar behavior, and the largest difference occurs for prismatic planes (at $H^{2}=0$, Figures $8(\mathrm{a})$ and (b)). To estimate the tension/compression asymmetry at the macroscopic level, the average (or equivalent) elastic strain $\langle\varepsilon\rangle$ (as proposed by Reference 46 ) was calculated as

$$
\langle\varepsilon\rangle=\frac{\sum_{h k l} \varepsilon_{h k l} f_{h k l}}{\sum_{h k l} f_{h k l}}
$$

where $f_{h k l}$ is the fraction of grains with orientation $\langle h k l\rangle$. This fraction depends on the direction inside the 


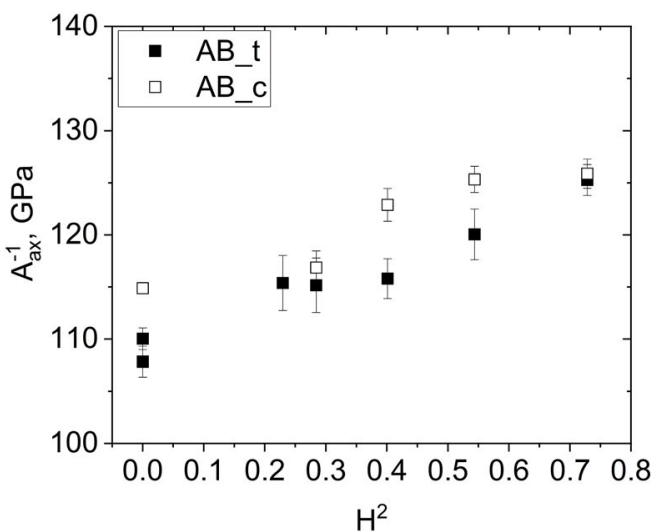

(a)

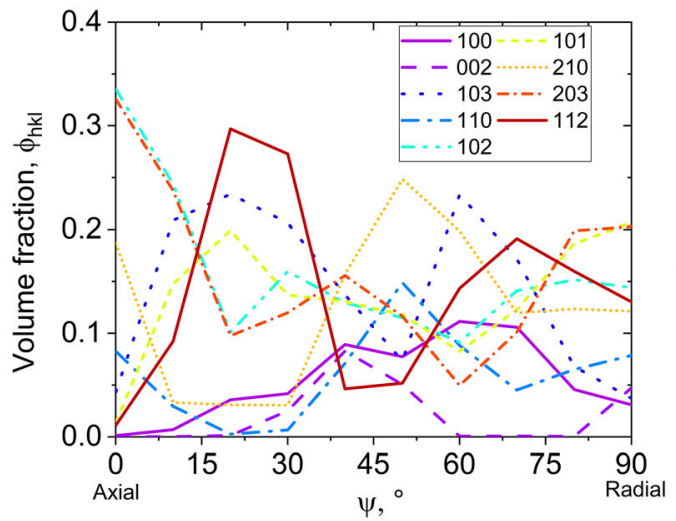

(c)

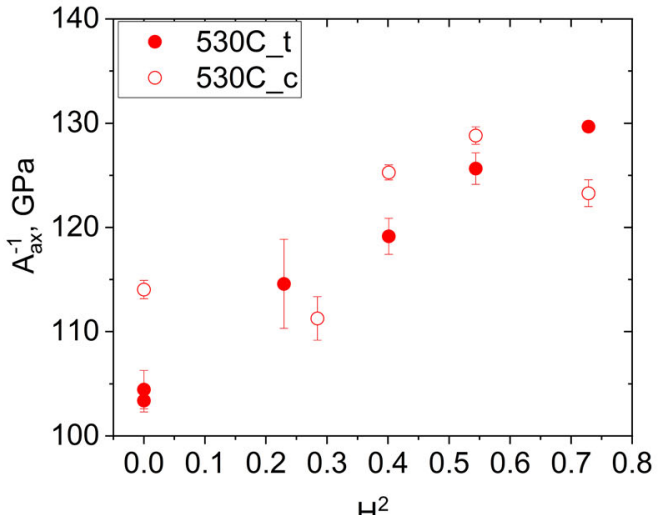

(b)

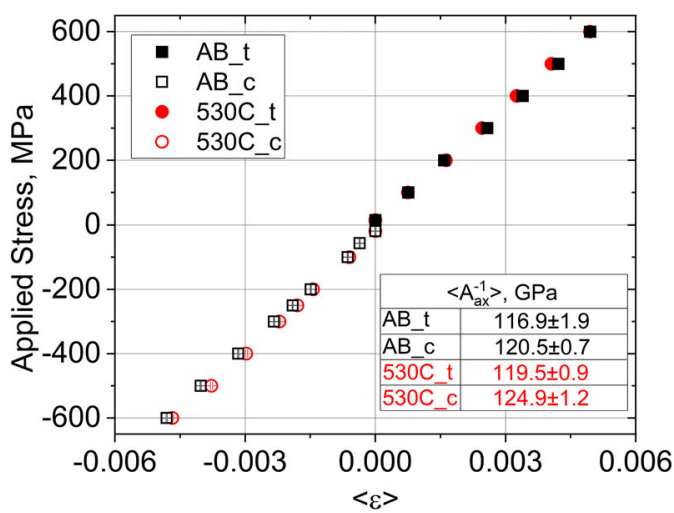

(d)

Fig. 8- The inverse of $S \mu E C T$ component $A_{\mathrm{ax}}^{-1}$ as a function of the orientation factor $H^{2}$ for $(a) \mathrm{AB}$ and $(b)$ for 530C. (c) Volume fraction of diffracting $h k l$ grains as a function of the azimuth angle $\psi$, and $(d)$ the applied stress as a function of mean lattice strain curves for tension and compression.

specimen, i.e., on the angle $\psi$ of the specimen inclination to the scattering vector. $f_{h k l}(\psi)$ can be extracted from the texture measurements (pole figures, Figure 5) using the texture intensity $\left(I_{h k l}(\psi)\right)$ of every reflection and weighting it by its multiplicity $m_{h k l}$ (note that nine peaks were used for this calculation):

$$
f_{h k l}(\psi)=\frac{I_{h k l}(\psi) m_{h k l}}{\sum_{h k l=1}^{9} I_{h k l}(\psi) \cdot m_{h k l}}
$$

Since the material presents a double fiber texture (Figure 5), the pole figures can be integrated along $\varphi$ and the ODF depends only on the angle $\psi$. This calculation yields the dependence of $f_{h k l}(\psi)$ on the tilt angle with respect to the sample long direction or, in other words, the azimuthal distribution of the fractions of a crystallite family from the axial to the radial directions (Figure $8(\mathrm{c})$ ). $f_{h k l}(0)$ is the necessary quantity for the calculation of the axial average lattice strain (Figure 8(d)).

Using Eq. [6], we obtain that the average $A_{\mathrm{ax}}^{-1}$ is higher for compression by around $5 \mathrm{GPa}$ for both $\mathrm{AB}$ and $530 \mathrm{C}$ samples (Figure 8(d)). One of the reasons for this difference can be defects and their orientation. As observed in Figure 3, and reported in the literature, ${ }^{[47-49]}$ LPBF materials often present a lack of fusion defects (crack-like) aligned perpendicular to the building direction (Figures 3(c) and (d)). During a compression test along the building direction, such defects are expected to (mechanically) close, while under tension, they should open and even propagate. A similar behavior is observed in microcracked ceramics. ${ }^{[50]}$ However, in that case, the crack density is much larger and the difference between the slopes of the stress-strain curves in tension and compression is more obvious than in the present study. According to CT images, the volume fraction of pores (within the $\mathrm{CT}$ resolution limit) reached only around 0.01 pct (Figure 3 ). While porosity and crack density are not directly linked (cracks have negligible volume), we can estimate the crack density to be low also in our LPBF Ti-6Al-4V samples.

\section{CONCLUSIONS}

The connection between macroscopic stress and diffraction (micro-) strain was made by the $S \mu E C T$, well known in micromechanics. We experimentally determined two components of the $S \mu E C T$ by in-situ SXRD in LPBF Ti-6Al-4V. We compared experimental 
data with the output of the classic models (Voigt, Reuss, and Kröner) used to calculate the so-called DECs. We concluded the following:

1. The usage of Voigt's model is equivalent to that of the macroscopic elastic constants, so that it should be avoided for diffraction-based residual stress determination in materials with high anisotropy.

2. A large difference of DECs (or axial $S \mu E C T A_{\mathrm{ax}}^{-1}$ ) between AM and wrought Ti-6Al-4V exists. This is attributed to texture and microstructural differences.

3. Acceptable agreement between experimental $A_{\mathrm{ax}}^{-1}$ and the Kröner or Reuss model (assuming isotropic microstructure) was found. However, a more appropriate model capturing microstructural features (e.g., grain size and shape) and texture should be developed.

4. In the case of complex or new materials, the experimental determination of $S \mu E C T$ is necessary.

5. A tension-compression asymmetry of both the microstrain and average macrostrain curves vs applied stress was observed and possibly attributed to the presence of the lack of fusion/crack-like defects, detected in computed tomography reconstructions.

Finally, for the sake of reproducibility and traceability of the results, we recommend explicit reporting of the DECs (or the $S \mu E C T$ components) used for residual stress calculations. Failing to do so would render it impossible to compare or benchmark different measurements (e.g., bulk or surface stress or studies of process parameter variation) and draw general conclusions about the residual stress state in LPBF parts.

\section{ACKNOWLEDGMENTS}

Open Access funding provided by Projekt DEAL. Christoph Genzel and Manuela Klaus (HZB, Berlin) are acknowledged for their support during the beamtime on EDDI.

\section{OPEN ACCESS}

This article is licensed under a Creative Commons Attribution 4.0 International License, which permits use, sharing, adaptation, distribution and reproduction in any medium or format, as long as you give appropriate credit to the original author(s) and the source, provide a link to the Creative Commons licence, and indicate if changes were made. The images or other third party material in this article are included in the article's Creative Commons licence, unless indicated otherwise in a credit line to the material. If material is not included in the article's Creative Commons licence and your intended use is not permitted by statutory regulation or exceeds the permitted use, you will need to obtain permission directly from the copyright holder. To view a copy of this licence, visit http://creat ivecommons.org/licenses/by/4.0/.

\section{REFERENCES}

1. J.L. Bartlett and X. Li: Addit. Manufact., 2019, vol. 27, pp. 131-49.

2. B. Vrancken, V. Cain, R. Knutsen, and J. Van Humbeeck: Scripta Mater., 2014, vol. 87, pp. 29-32.

3. P. Mercelis and J.-P. Kruth: Rap. Prototyp. J., 2006, vol. 12, pp. 254-65.

4. T. Thiede, S. Cabeza, T. Mishurova, N. Nadammal, A. Kromm, J. Bode, C. Haberland, and G. Bruno: Mater. Perfor. Charact., 2018 , vol. 4 , pp. $717-35$.

5. T. Mishurova, S. Cabeza, K. Artzt, J. Haubrich, M. Klaus, C. Genzel, G. Requena, and G. Bruno: Materials, 2017, vol. 10, pp. 348-62.

6. T. Mishurova, S. Cabeza, T. Thiede, N. Nadammal, A. Kromm, M. Klaus, C. Genzel, C. Haberland, and G. Bruno: Metall. Mater. Trans. A, 2018, vol. 49A, pp. 3038-46.

7. A. Khorasani, I. Gibson, M. Goldberg, and G. Littlefair: Rap. Prototyp. J., 2017, vol. 23, pp. 295-304.

8. H. Shipley, D. McDonnell, M. Culleton, R. Coull, R. Lupoi, G. O'Donnell, and D. Trimble: Int. J. Mach. Tools Manuf., 2018, vol. 128 , pp. 1-20.

9. J. Haubrich, J. Gussone, P. Barriobero-Vila, P. Kürnsteiner, E.A. Jägle, D. Raabe, N. Schell, and G. Requena: Acta Mater., 2019, vol. 167, pp. 136-48.

10. T. Mishurova, K. Artzt, J. Haubrich, G. Requena, and G. Bruno: Addit. Manuf., 2019, vol. 25, pp. 325-34.

11. V. Hauk: Structural and Residual Stress Analysis by Nondestructive Methods, Elsevier Science B.V., Amsterdam, 1997.

12. E. Maawad, H.G. Brokmeier, Z.Y. Zhong, N. Al-Hamdany, M. Salih, L. Wagner, and N. Schell: Mater. Sci. Eng. A, 2014, vol. 594 , pp. $62-67$.

13. A. Cervellino, P.M. Derlet, and H. Van Swygenhoven: Acta Mater., 2006, vol. 54, pp. 1851-56.

14. G. Bruno and B.D. Dunn: Measur. Sci. Technol., 1997, vol. 8, pp. $1244-49$.

15. T. Voisin, N.P. Calta, S.A. Khairallah, J.-B. Forien, L. Balogh, R.W. Cunningham, A.D. Rollett, and Y. Morris Wang: Mater. Des., 2018, vol. 158, pp. 113-26.

16. N. Dumontet, G. Geandier, F. Galliano, B. Viguier, and B. Malard: Residual Stresses 2018-ECRS-10, Materials Research Proceedings, Leuven, Belgium, 2018, pp 289-94.

17. A.M. Stapleton, S.L. Raghunathan, I. Bantounas, H.J. Stone, T.C. Lindley, and D. Dye: Acta Mater., 2008, vol. 56, pp. 6186-96.

18. N. Nadammal, S. Cabeza, T. Mishurova, T. Thiede, A. Kromm, C. Seyfert, L. Farahbod, C. Haberland, J.A. Schneider, P.D. Portella, and G. Bruno: Mater. Des., 2017, vol. 134, pp. 139-50.

19. S. Gorsse, C. Hutchinson, M. Goune, and R. Banerjee: Sci. Technol. Adv. Mater., 2017, vol. 18, pp. 584-610.

20. A. Pesach, E. Tiferet, S.C. Vogel, M. Chonin, A. Diskin, L. Zilberman, O. Rivin, O. Yeheskel, and E.N. Caspi: Addit. Manufact., 2018, vol. 23, pp. 394-401

21. M. Simonelli, Y.Y. Tse, and C. TuckSimonelli: Metall. Mater. Trans. A, 2014, vol. 45A, pp. 2863-72.

22. P.E. Aba-Perea, T. Pirling, P.J. Withers, J. Kelleher, S. Kabra, and M. Preuss: Mater. Des., 2016, vol. 89, pp. 856-63.

23. U. Welzel and E.J. Mittemeijer: J. Appl. Phys., 2003, vol. 93, pp. 9001-11.

24. S. Matthies, H.G. Priesmeyer, and M.R. Daymond: J. Appl. Crystallogr., 2001, vol. 34, pp. 585-601.

25. M.F. Slim, A. Alhussein, E. Zgheib, and M. François: Acta Mater., 2019, vol. 175, pp. 348-60.

26. A. Heldmann, M. Hoelzel, M. Hofmann, W. Gan, W.W. Schmahl, E. Griesshaber, T. Hansen, N. Schell, and W. Petry: J. Appl. Crystallogr., 2019, vol. 52, pp. 1144-56.

27. T. Thiede, T. Mishurova, S. Evsevleev, I. Serrano-Munoz, C. Gollwitzer, and G. Bruno: Quant. Beam Sci., 2019, vol. 3, p. 3.

28. T. Mishurova, K. Artzt, J. Haubrich, G. Requena, and G. Bruno: Metals, 2019, vol. 9, p. 261. 
29. G. Kasperovich, J. Haubrich, J. Gussone, and G. Requena: Mater. Des., 2016, vol. 105, pp. 160-70.

30. C. Genzel, I.A. Denks, J. Gibmeier, M. Klaus, and G. Wagener: Nucl. Instrum. Meth. Phys. Res. Sect. A: Acceler. Spectrom. Detec. Assoc. Equip., 2007, vol. 578, pp. 23-33.

31. D. Apel, M. Meixner, A. Liehr, M. Klaus, S. Degener, G. Wagener, C. Franz, W. Zinn, C. Genzel, and B. Scholtes: Nucl. Instrum. Meth. Phys. Res. Sect. A: Acceler. Spectrom. Detect. Assoc. Equip., 2018, vol. 2018 (877), pp. 56-64.

32. K. Pawlik and P. Ozga: Goett. Arb. Geol. Palaeontol., 1999, vol. SB4, pp. 146-47.

33. Avizo Software, Thermo Fisher Scientific, https://www.fei.com/sof tware/avizo, accessed 03.092019.

34. W. Xu, M. Brandt, S. Sun, J. Elambasseril, Q. Liu, K. Latham, K. Xia, and M. Qian: Acta Mater., 2015, vol. 85, pp. 74-84.

35. P. Barriobero-Vila, J. Gussone, J. Haubrich, S. Sandlöbes, J. Da Silva, P. Cloetens, N. Schell, and G. Requena: Materials, 2017, vol. 10, p. 268.

36. S. Tammas-Williams, P.J. Withers, I. Todd, and P.B. Prangnell: Sci. Rep., 2017, vol. 7, p. 7308.

37. A.M. beese and B.E. Carroll: JOM, 2015, vol. 68, pp. 724-34.

38. W. Te Tai: Int. J. Sol. Struct., 1966, vol. 2, pp. 1-8.

39. H. Wern, R. Johannes, and H. Walz: Phys. Status Solidi (b), 1998, vol. 206, pp. 545-57.

40. T. Gnäupel-Herold, A.A. Creuziger, and M. Iadicola: J. Appl. Crystallogr., 2012, vol. 45, pp. 197-206.
41. H. Wern: XEC, Hochschule für Technik und Wirtschaft Saarbrücken, Saarbrücken, Germany, 2000.

42. H. Dölle: J. Appl. Crystallogr., 1979, vol. 12.

43. M. Simonelli, Y.Y. Tse, and C. Tuck: Mater. Sci. Eng. A, 2014, vol. 616 , pp. 1-11.

44. VT Em, SY Ivanov, ID Karpov, SA Rylov, EV Zemlyakov, and KD Babkin: J. Phys. Conf. Ser., 2018, https://doi.org/10.1088/ 1742-6596/1109/1/012049.

45. D. Faurie, P. Djemia, E. Le Bourhis, P.O. Renault, Y. Roussigné, S.M. Chérif, R. Brenner, O. Castelnau, G. Patriarche, and P. Goudeau: Acta Mater., 2010, vol. 58, pp. 4998-5008.

46. M.R. Daymond: J. Appl. Phys., 2004, vol. 96, pp. 4263-72.

47. I. Maskery, N.T. Aboulkhair, M.R. Corfield, C. Tuck, A.T. Clare, R.K. Leach, R.D. Wildman, I.A. Ashcroft, and R.J.M. Hague: Mater. Charact., 2016, vol. 111, pp. 193-204.

48. R. Laquai, B.R. Müller, G. Kasperovich, J. Haubrich, G. Requena, and G. Bruno: Mater. Res. Lett., 2017, vol. 6, pp. $130-35$.

49. G. Dongdong, Y.-C. Hagedorn, W. Meiners, G. Meng, R.J.S. Batista, K. Wissenbach, and R. Poprawe: Acta Mater., 2012, vol. 60, pp. 3849-60.

50. G. Bruno and M. Kachanov: J. Eur. Ceram. Soc., 2013, vol. 33, pp. 2073-85.

Publisher's Note Springer Nature remains neutral with regard to jurisdictional claims in published maps and institutional affiliations. 